\title{
Kink-induced transport and segregation in oscillated granular layers
}

\author{
Sung Joon Moon $*$ Daniel I. Goldman, J. B. Swift 7 and Harry L. Swinney \\ Center for Nonlinear Dynamics and Department of Physics, University of Texas, Austin, Texas 78712
}

(Dated: June 12, 2018)

\begin{abstract}
We use experiments and molecular dynamics simulations of vertically oscillated granular layers to study horizontal particle segregation induced by a kink (a boundary between domains oscillating out of phase). Counter-rotating convection rolls carry the larger particles in a bidisperse layer along the granular surface to a kink, where they become trapped. The convection originates from avalanches that occur inside the layer, along the interface between solidified and fluidized grains. The position of a kink can be controlled by modulation of the container frequency, making possible systematic harvesting of the larger particles.
\end{abstract}

PACS numbers: $45.70 . \mathrm{Mg}, 81.05 . \mathrm{Rm}, 44.27 .+\mathrm{g}, 05.60 .-\mathrm{k}$

In 1831 Faraday observed convective motion of grains in heaps in vertically oscillated granular layers [1]. Later there were many studies of convection in oscillated granular materials $[2,3,4,4,6,6,8,6,10,11,12$. These convection phenomena are driven by interstitial air [5, 6] and shear due to sidewalls [7, 10, 11]. One consequence of the sidewall-driven convection is vertical segregation of grains of different sizes, which is an example of the Brazil-nut effect 13, 14, 15.

We consider here a different kind of convection, one that is driven not by interstitial air or sidewalls but rather arises from the intrinsic dynamics of the oscillated granular layers. This convection is associated with kinks, which are boundaries separating oscillating domains of opposite phase; when the layer on one side of a kink is moving up, the layer on the other side of the kink is moving down. Kinks can form in oscillating layers when the maximum container acceleration $a_{\max }$ is large enough $\left(a_{\max } \geq 4.5 g\right.$, where $g$ is the gravitational acceleration) so that the granular layer hits the container bottom only every other cycle [16, 17]. Kinks spontaneously form in a layer for $a_{\max }>7 g[18]$.

During each oscillation cycle, the granular material on one side of a kink strikes the container bottom and solidifies, while the fluidized grains on the other side avalanche down along the front of the solidified grains. We examine this avalanche process, which will be shown to be responsible for convection associated with kinks. In a bidisperse layer, such convective motion conveys larger grains toward the kink. We will show that controlled motion of a kink leads to horizontal size segregation, in contrast to the vertical size segregation in the Brazil-nut effect. We will first describe the laboratory observations, and then show how the particle transport, trapping, and horizontal segregation can be understood using molecular dynamics simulations.

Experimental observations: Trapping, transport, and segregation - We find that larger grains in the vicinity of a kink in an oscillating bidisperse layer move toward the kink and remain there, while the larger grains far from a kink move only diffusively, as the solid white lines (a)
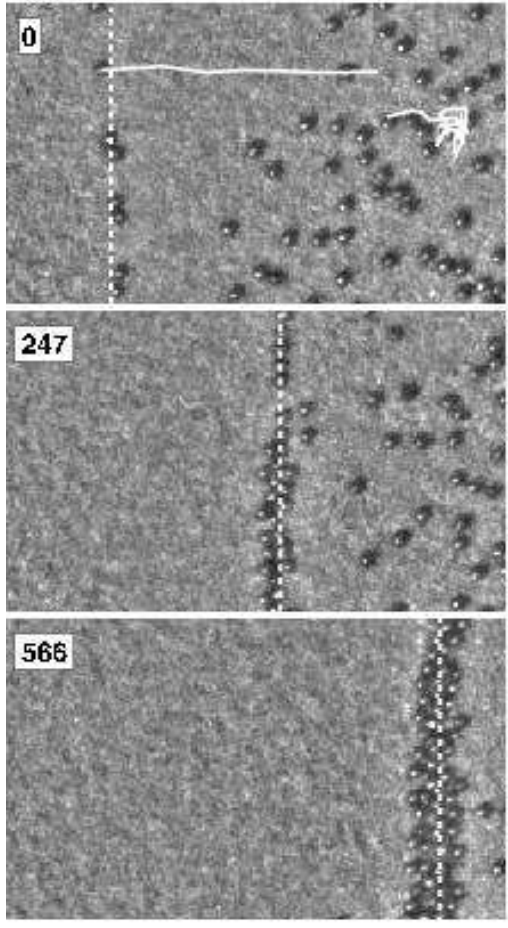

(b)

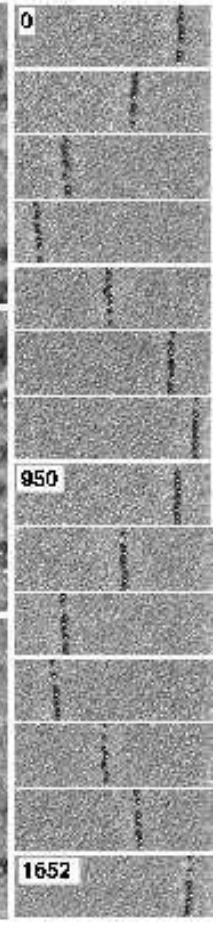

FIG. 1: Segregation and controlled transport of $650 \mu \mathrm{m}$ diameter black glass spheres by a kink in an experiment with a 10-particle deep oscillated layer of $165 \mu \mathrm{m}$ bronze spheres at $a_{\max }=5 g$ and $f=92 \mathrm{~Hz}$. The motion of the kink is controlled by modulation of the phase difference $\Delta \phi$ between the primary oscillation signal and an added small subharmonic sinusoidal perturbation (see text). (a) A kink (dashed line) sweeps across the layer after a rapid change in $\Delta \phi$ of $2 \pi / 3$; glass spheres close to the kink move toward it and remain trapped in the kink at the surface of the layer, while far from the kink they diffuse; these two types of trajectories are illustrated by the solid white lines. The numbers in each frame denote plate oscillations after $\Delta \phi$ is changed. (b) Sinusoidal oscillation of a kink, with $\Delta \phi(t)=f_{m s} / f_{m r} \sin \left(2 \pi f_{m r} t\right)$, $f_{m s}=0.17 \mathrm{~Hz}$ and $f_{m r}=0.1 \mathrm{~Hz}$. The large trapped particles follow the motion of the kink. 

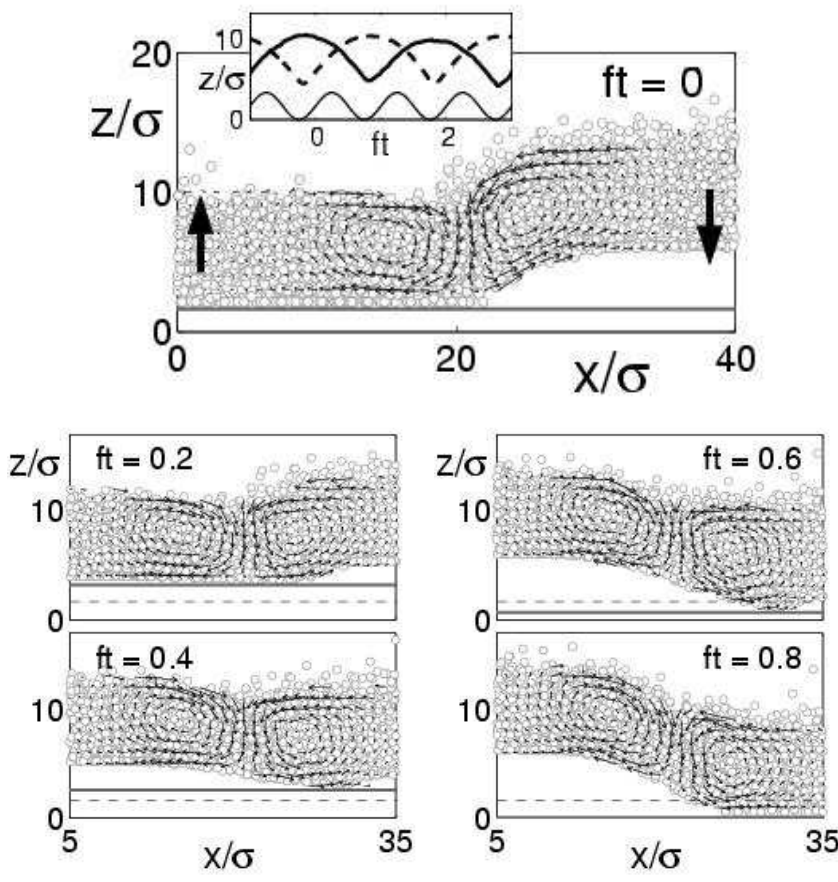

FIG. 2: Grains form a pair of convection rolls, flowing downward at the kink, as illustrated by these projections of a layer with a kink at different times $f t$ during a cycle $(f t=0$ when the container is at its equilibrium position, moving upward), obtained from a simulation $\left(a_{\max }=5.2 g, f=69 \mathrm{~Hz}\right)$. The vertical position of the center of mass as a function of time of the left (right) side of the kink is shown as dashed (solid) line in the inset of the top panel. The two bold vertical arrows on either side of the kink indicate the directions of motion of these two domains. The small arrows show the discrete grain velocity $\mathbf{u}(\mathbf{r}(t), t)$ averaged over the shorter horizontal direction. Each circle represents a grain, and the container bottom is indicated by horizontal gray lines. The region shown is far from the rigid sidewalls in the longer horizontal direction.

in Fig. 1 (a) illustrate. Further, if the location of a kink is controlled by modulating the container oscillation frequency [19], the large grains follow the motion of the kink (Fig. 1 (b)); this leads to a horizontal size segregation. We consider a 10-particle deep layer of $165 \mu \mathrm{m}$ bronze spheres (mass density $8.3 \mathrm{~g} / \mathrm{cm}^{3}$ ) together with a few hundred of $650 \mu \mathrm{m}$ glass spheres $\left(2.5 \mathrm{~g} / \mathrm{cm}^{3}\right)$, in an evacuated square container of horizontal area $8.9 \times 8.9 \mathrm{~cm}^{2}$ (the pressure is $5 \mathrm{~Pa}$ ). The layer is subject to a vertical sinusoidal oscillation with an amplitude $A$ and a frequency $f$; the oscillation is characterized by two of the following three parameters: the maximum acceleration $a_{\max }=A(2 \pi f)^{2}$, the maximum velocity $V_{\max }=2 \pi A f$, and the frequency $f$. In some experiments we add a perturbative subharmonic forcing to control the position of a kink, where the plate position $z$ is given by $z(t)=A \sin (2 \pi f t)+0.01 A \sin (\pi f t+\Delta \phi(t))$. This secondary forcing breaks the symmetry of the momentum transfer across the kink, which makes the kink move [18].
The relative phase between the main oscillation and the subharmonic perturbation controls the position of the kink within the container (see Fig. 11).

We consider the range $4.5 g<a_{\max }<5.5 g$, where the layer remains flat except for the kinks; similar transport and segregation occur for larger $a_{\max }$ where wave patterns form, but it is simpler to quantify the transport and segregation processes for a non-patterned flat layer.

Microscopic dynamics; kink-associated convection The experimental observations reveal that a mean flow toward a kink exists in the vicinity of the kink. We use a previously validated three-dimensional (3-d) molecular dynamics simulation [18, 20], to understand the trapping and transport produced by the microscopic dynamics. The coefficient of restitution $e$ and the coefficient of friction $\mu$ were set to 0.7 and 0.5 respectively, as in Ref. 20]. We simulate 8-particle deep monodisperse layers of spherical grains of diameter $165 \mu \mathrm{m}$ in a rectangular box of horizontal area $160 \sigma \times 10 \sigma$, where $\sigma$ is the particle diameter. Periodic boundary conditions are used in the shorter horizontal direction ( $y$-axis), and vertical rigid sidewalls are imposed in the longer horizontal direction ( $x$-axis). The rigid sidewalls and the bottom plate are assumed to be made of the same material as grains.

We distinguish two types of velocities, the instantaneous grain velocity $\mathbf{v}(\mathbf{r}(t), t)=\lim _{\Delta t \rightarrow 0^{+}}(\mathbf{r}(t+\Delta t)-$ $\mathbf{r}(t)) / \Delta t$ and the discrete grain velocity $\mathbf{u}(\mathbf{r}(t), t)=$ $(\mathbf{r}(t+2 T)-\mathbf{r}(t)) /(2 T)$, where $\mathbf{r}(t)$ is the position vector of a grain at a given time $t$, and $2 T=2 / f$ is the oscillation period of the macroscopic state. The discrete velocity corresponds to the grain velocity in the $f / 2$-strobed frame. Following the trajectories of individual grains, we observe that grains do not come back to the same positions after $2 T$, even though the layer returns to the same macroscopic state. Instead, in the discrete velocity field, grains form a pair of counter-rotating convection rolls that flow downward at the kink (Fig. 2). The shape of the rolls changes during a cycle, as does the shape of the

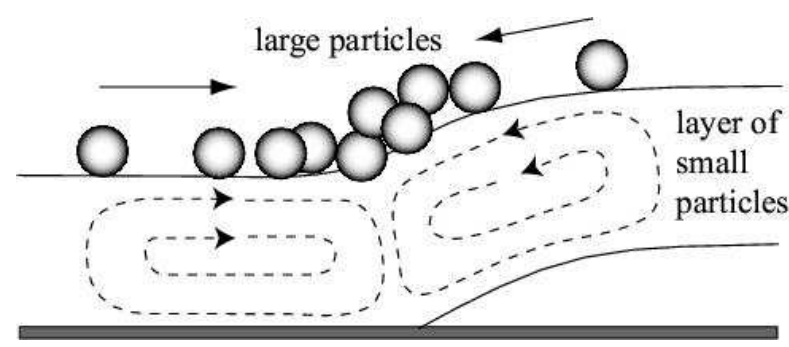

FIG. 3: A schematic diagram showing trapping of larger particles at a kink in a layer of smaller particles. The larger particles move toward the kink due to the surface flow of the convective motion of the smaller particles. The larger particles do not flow down in between the convection rolls, but remain there, due to their size. Dashed arrows represent the convection rolls formed by the smaller particles, and solid arrows indicate the direction of the motion of large particles. 


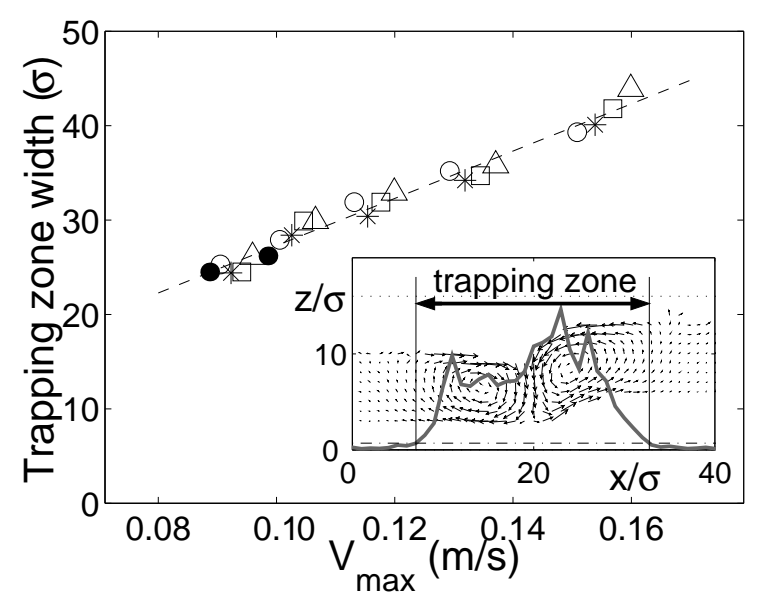

FIG. 4: The trapping zone width (defined in the inset) for various $a_{\max }$ 's and $f$ 's, obtained in simulations of 8-particle deep monodisperse layers of $165 \mu \mathrm{m}$ spheres. The width is linearly proportional to $V_{\max }$, as shown by the dashed line (a least square fit). The symbols correspond to: $\bullet, a_{\max }=4.9 \mathrm{~g}$; $\circ, 5 g ; *, 5.1 g ; \square, 5.2 g ; \triangle, 5.3 g$. For each $a_{\max }$, the data points correspond to $f=86 \mathrm{~Hz}, 78 \mathrm{~Hz}, 69 \mathrm{~Hz}, 60 \mathrm{~Hz}, 52$ $\mathrm{Hz}$, respectively. Inset: The trapping zone is defined as the region where the vertically integrated $|\mathbf{u}|^{2}$ (gray solid line, in an arbitrary unit) is larger than some small value (dash-dotted line).

layer, but the sense of circulation remains the same. For the parameters of Fig. 22 it takes about $100 \mathrm{~T}$ for grains in the outer-most part of the rolls to complete a full circuit.

Trapping and transport - The presence of the convection rolls explains the motion of large grains toward a kink: Large grains far from the kink rise to the surface as a consequence of the Brazil-nut effect and those that enter the convecting region will move toward the kink due to the surface flow of the convective motion (Fig. 31). When the large grain reaches the down-flow channel region between the convection rolls, it will not flow down because it is too large, and it becomes trapped there, as illustrated in Fig. 1] We have observed trapping for a wide range of impurity particle diameters and densities relative to those of the oscillated bronze spheres. We introduced hundreds of the same type of impurity particles, including lead, stainless steel, glass, and polystyrene spheres of diameter ratios 1.12 to 11.8 , density ratios 0.14 to 1.4 , and mass ratios 0.4 to 2500 . Trapping occurred in all cases studied except for the diameter ratio below 1.12 , where the motion of the impurity grains was visually indistinguishable from the motion of the bronze spheres.

We refer to a region in which large grains move toward a kink and become trapped as the "trapping zone". The width of the trapping zone, defined in Fig. 4 is found to be linearly proportional to $V_{\max }$ for the range of oscillation parameters in our study (Fig. 4). There is a limit to the number of large grains that can be trapped; for (a) Lab. frame
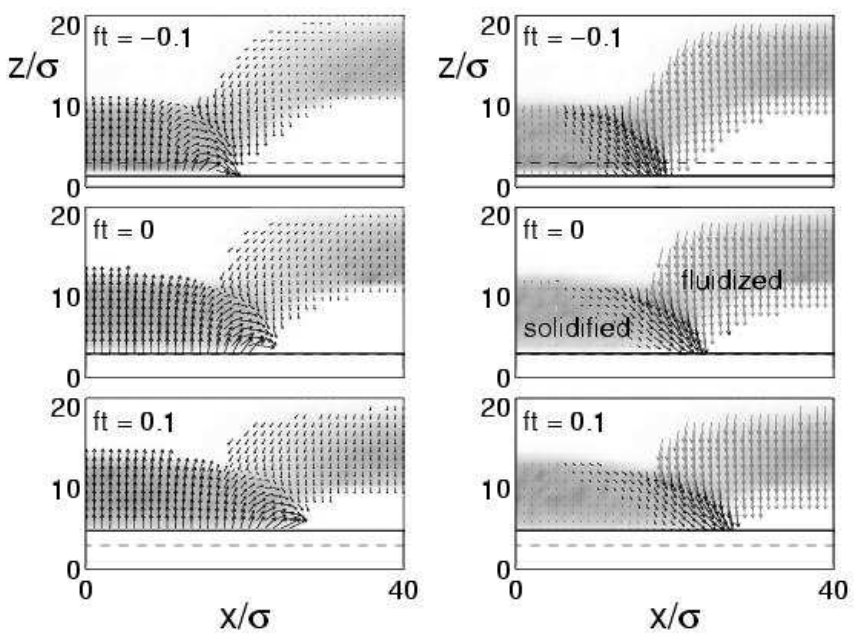

FIG. 5: Simulations reveal an avalanche occurring inside the layer, along the interface between the solidified and fluidized parts: (a) Instantaneous grain velocities $\mathbf{v}(\mathbf{r}(t), t)$ in the laboratory frame (indicated by arrows), averaged over the shorter horizontal direction, when the left side of a kink is being pushed up by the bottom plate (a horizontal solid line) for the same case as in Fig. 2 A gray background represents the layer. (b) Instantaneous velocities in the container frame, during the same time. As the left side is pushed up, it solidifies on the container bottom, while the right side is fluidized and cascades down onto the left side, as shown by gray arrows. $\left(a_{\max }=5.2 g, f=69 \mathrm{~Hz}\right)$.

the range of $V_{\max }$ studied in this paper, the maximum number of trapped particles corresponds to the number of large grains forming a monolayer above the trapping zone. In the case of Fig. 1 (a), $V_{\max }$ of the primary oscillation is $0.087 \mathrm{~m} / \mathrm{s}$, and the trapping zone width from the last frame $(t=566 T)$ is seen to be about 5 glass particle diameters or 20 bronze particle diameters, which

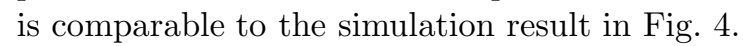

As $V_{\max }$ increases, qualitative changes occur in the motion of large grains: The down-flow channel region dilates more (i.e., the granular volume fraction decreases) so that there is enough room for large grains to circulate with the convecting smaller grains throughout the volume of the trapping region, disappearing in the kink and popping up from the upward flow region of the convection rolls. The maximum number of trapped particles in this regime is not determined by the area of the trapping zone; we have not investigated this regime.

We observe in the experiment that when a kink moves too rapidly, large grains leak out of the kink; there is a maximum speed of a kink for which it can convey large grains. The mechanism in Fig. 3 suggests that if a kink moves more than the trapping zone width during a cycle, the large grains would not follow the motion; the experimental observations are consistent in that when the speed of a kink exceeds approximately twice the layer 
depth (comparable to the zone width in Fig. 1) per time $2 T$, large grains are left behind the kink.

Internal avalanches and convection - An examination of the instantaneous velocity field $\mathbf{v}(\mathbf{r}(t), t)$ reveals that the convection is driven by avalanches oscillating perpendicular to the kink. When the left side of a kink is pushed up, the right side cascades down onto the left side of the kink (Fig. 5 (a)). These avalanches inside the layer are visualized better when the velocity field is viewed in the container frame (Fig. 5] (b)): When the left side is pushed up, it solidifies and moves with the same velocity as the container until it takes off from the container. Meanwhile, the right side is still falling and is fluidized. This leads to a density gradient between the solidified and the fluidized regions. Grains flow along the surface of the solidified region, toward the right side. We refer to this motion inside the layer as an internal avalanche, to contrast with the usual avalanche, which occurs on a surface 21]. (Such an internal avalanche was suggested by Laroche et al. [5] as a mechanism for convection in heaping.) In the following cycle, another internal avalanche occurs in the opposite direction, pushing grains back to the left side and to lower heights. The oscillatory internal avalanches result in the downward flow at the kink. At impact with the container, grains at the bottom of the layer move away from the kink in a horizontal direction and later rise, forming the upward flow at the outer side of the rolls. The trapping zone width (or convection roll size) is determined by the horizontal displacements of such grains.

The convective motion that has been observed previously in subharmonic standing wave patterns in oscillated layers 22] can be explained by the same mechanism: when a patterned layer collides with the container, large density gradients form along the pattern inside the layer. Internal avalanches perpendicular to these gradients lead to convection rolls.

Conclusions - We have described a form of convection in granular media that is driven not by interaction of the grains with air or the container sidewalls but by the intrinsic dynamics of the layer when a kink is present. The kink and internal avalanches that drive this convective motion are unique to oscillating granular media because the solidification and fluidization during each cycle that occur in the granular layer do not occur in an oscillated liquid layer.

The kinks that lead to the avalanche motion and convection are ubiquitous in oscillated granular layers at high enough container accelerations $\left(a_{\max }>7 g\right)$; hence kink-associated convection and trapping of large particles are generic features of bidisperse oscillated granular layers in that regime. Since the location of a kink can be controlled by modulation of the container oscillation frequency, it is possible to harvest the segregated larger particles in a controlled way by sweeping a kink to the edge of a container.

This work was supported by the Engineering Research Program of the Office of Basic Energy Sciences of the U. S. Department of Energy (Grant No. DE-FG0393ER14312) and The Texas Advanced Research Program (Grant No. ARP-055-2001).

* moon@chaos.utexas.edu

† swift@chaos.utexas.edu

[1] M. Faraday, Phil. Trans. R. Soc. London 52, 299 (1831).

[2] W. Kroll, Chem. Ingr. Tech. 27, 33 (1955).

[3] G. Rátkai, Powder Technol. 15, 187 (1976).

[4] S. B. Savage, J. Fluid Mech. 194, 457 (1988).

[5] C. Laroche, S. Douady, and S. Fauve, J. Phys. France 50, 699 (1989).

[6] H. K. Pak, E. Van Doorn, and R. P. Behringer, Phys. Rev. Lett. 74, 4643 (1995).

[7] E. Clément, J. Duran, and J. Rajchenbach, Phys. Rev. Lett. 69, 1189 (1992).

[8] J. A. C. Gallas, H. J. Herrmann, and S. Sokołowski, Phys. Rev. Lett. 69, 1371 (1992).

[9] Y-h. Taguchi, Phys. Rev. Lett. 69, 1367 (1992).

[10] J. Lee, J. Phys. A 27, L257 (1994).

[11] J. B. Knight, H. M. Jaeger, and S. R. Nagel, Phys. Rev. Lett. 70, 3728 (1993); E. E. Ehrichs, H. M. Jaeger, G. S. Karczmar, J. B. Knight, V. Yu. Kuperman, and S. R. Nagel, Science 267, 1632 (1995); J. B. Knight, Phys. Rev. E 55, 6016 (1997); E. L. Grossman, ibid. 56, 3290 (1997).

[12] R. D. Wildman, J. M. Huntley, J.-P. Hansen, D. J. Parker, and D. A. Allen, Phys. Rev. E 62, 3826 (2000); R. D. Wildman, J. M. Huntley, and D. J. Parker, Phys. Rev. Lett. 86, 3304 (2001).

[13] R. L. Brown, J. Inst. Fuel 13, 15 (1939).

[14] J. C. Williams, Powder Technol. 15, 245 (1976).

[15] M. E. Möbius, B. E. Lauderdale, S. R. Nagel, and H. M Jaeger, Nature, 414, 270 (2001).

[16] F. Melo, P. B. Umbanhowar, and H. L. Swinney, Phys. Rev. Lett. 75, 3838 (1995).

[17] P. B. Umbanhowar, F. Melo, and H. L. Swinney, Physica A 249, 1 (1998).

[18] S. J. Moon, M. D. Shattuck, C. Bizon, D. I. Goldman, J. B. Swift, and H. L. Swinney, Phys. Rev. E 65011301 (2002).

[19] I. S. Aranson, D. Blair, W. K. Kwok, G. Karapetrov, U. Welp, G. W. Crabtree, V. M. Vinokur, and L. S. Tsimring, Phys. Rev. Lett. 82, 731 (1999).

[20] C. Bizon, M. D. Shattuck, J. B. Swift, W. D. McCormick, and H. L. Swinney, Phys. Rev. Lett. 80, 57 (1998).

[21] J. Rajchenbach, Phys. Rev. Lett. 89, 074301 (2002).

[22] C. Bizon, M. D. Shattuck, J. R. de Bruyn, J. B. Swift, W. D. McCormick, and H. L. Swinney, J. Stat. Phys. 93, 449 (1998). 